\title{
Selection of students to medical school
}

Selection criteria must be based on valid predictors of performance in medical school

A recent leading article in the Ceylon Medical Journal that deals with an important policy issue emphasizes that the ultimate goal of selection of students to a medical school must be to identify those who are likely to contribute towards meeting the health needs of society [1]. In discussing the pros and cons of the district quota system used for the selection of medical students in Sri Lanka since the 1970s, this leading article mentions the need for studies that examine the correlation between admission criteria and medical school performance.

We have recently carried out several such studies. The first showed that although the raw aggregate score from the GCE Advanced Level examination did show a statistically significant correlation with outcome (three summative examinations conducted in the medical school) at Ragama, it was a weak predictor of performance [2]. The second study, which extended the scope of the analysis to all six medical schools in the country, confirmed this finding [3]. It also showed that students who sat the A Level examination as school candidates (in their 1st or 2nd attempt at the examination) did much better in medical school than private candidates (who were probably in their $3 \mathrm{rd}$ attempt).

The leading article [1] also stresses the need for subject-wise standardisation, with Z-scores, rather than raw aggregate scores, as the basis for selection. This change came into effect in Sri Lanka in 2000, along with a change of subjects. The first intake of students selected according to Z-scores has not yet graduated from medical school, so a comprehensive analysis is not possible. However, we have just completed a correlational study similar to the two mentioned previously, using performance at the first summative examination in medical school as the outcome measure.

The study sample comprised medical students in Colombo $(n=299)$ and Ragama ( $n=196)$, entering university on the basis of their A Level examination results in $2001(n=195)$ and $2002(n=300) .70 .1 \%$ of these students passed the first examination in medical school at the first attempt; $35.6 \%$ with honours. We examined the predictive value of the $\mathrm{Z}$-score in determining examination outcome, using logistic regression in a model very similar to that described previously [3]. We found that although the Z-score was significantly associated with exam outcome, both for passing and obtaining honours, it explained only $4 \%$ of the variation in outcome in passing the examination at first attempt, and $5 \%$ of the variation in obtaining honours. The equivalent values for the aggregate score were $2 \%$ and $7 \%$ [3]. Thus it appears that the change from using raw aggregate scores to Z-scores may not have improved matters a great deal for selecting better medical students, perhaps because the subject combination has remained unchanged. Also, valid use of the $\mathrm{Z}$-score depends on the assumption that 
the shape of score distribution is similar in the different subjects. If that assumption is not valid here, it may be why the predictive validity of the A Level has not been increased by using the Z-score. An alternative method of standardisation that does not require the marks in different subjects to be similarly distributed would be a score derived from simply ranking students in each subject.

All these findings support the conclusion that the Sri Lankan A Level examination results are not, on their own, a strong predictor of the academic performance of students in medical school. Thus the advisability of doing away with the district quota, and replacing it with a selection process that is based solely on A level Z-scores, is debatable. Policy makers at the University Grants Commission and the Ministry of Education need to take serious note of Prof Bandaranayake's comment [1] that the process of selection must be determined by the nature of the curriculum in medical school, rather than changing the curriculum to cope with an inappropriate selection process. As we have said previously, significant improvement in the quality of medical graduates through a change in selection policy will require a change in the tools used for selection of students [3]. Until then, restricting the number of attempts at the selection examination to two, and increasing the merit quota (i.e., the proportion of students selected solely on all-island rank order of Z-scores) may bring about limited improvements.

\section{References}

1. Bandaranayake RC. Selection of students to medical school. Ceylon Medical Journal 2005; 50: 41-3.

2. de Silva NR, Pathmeswaran A, de Silva HJ. Selection of students for admission to a medical school in Sri Lanka. Ceylon Medical Journal 2004; 49: 81-5.

3. de Silva NR, Pathmeswaran A, de Silva N, Edirisinghe JS, Parameswaran SV, et al. Admission to medical schools in Sri Lanka: predictive validity of selection criteria. Ceylon Medical Journal 2006; 51: 17-21.

NR de Silva, Co-ordinator, Medical Education, MN Chandratilake, Medical Education Centre, and A Pathmeswaran, Department of Community Medicine, Faculty of Medicine, University of Kelaniya. R Dias, Director, Medical Education and Research Centre, Faculty of Medicine, University of Colombo.

Correspondence: NRdeS, e-mail: <nrdes@sltnet.lk> (Competing interests: none declared).

\section{Medical professionalism is not the Medical Councils' documents}

The assumption is that the meaning of medical professionalism lies in the General Medical Council's Good Medical Practice. It does not. Good Medical Practice is a seriously deficient document, and it is likely to continue to be so even though it is currently undergoing revision. Good Medical Practice sets out the duties that a doctor must discharge. Those duties are rules that are linked to punishment (removal from the medical register) if they are broken or breached. The word "professionalism" appears throughout Good Medical Practice as a comforting adjective. It lends support to words such as "competence" and "knowledge". But the notion of professionalism itself is ignored. The listed duties are not put in the context of a rigorously worked out view of what medical professionalism means. Put simply, the GMC has missed the significance of professionalism. This lapse is currently proving damaging to the credibility of the organisation.

The Royal College of Physicians Working Party on Medical Professionalism. [See: Horton R. Medicine: the prosperity of virtue. The Lancet 2005; 366: 1985-7.] See also page 73. 